\title{
Hábitos de lectura y su influencia en el aprendizaje de estudiantes de primaria
}

\author{
Melquiades Cordova Ludeña \\ melqiadescordova@gmail.com \\ https://orcid.org/0000-0002-5223-5763 \\ Universidad Cesar Vallejo. Lima - Perú \\ Teobaldo Carlos Sevilla Muñoz \\ Carlossevillam67@gmail.com \\ https://orcid.org/0000-0002-5468-9400 \\ Universidad Cesar Vallejo. Lima - Perú \\ Wilder Muñoz Aliaga \\ wilder.aliaga@gmail.com \\ https://orcid.org/0000-0002-2943-5783 \\ U.C. P. Enrique José Varona. La Habana - Cuba \\ Eufemia María Martínez Rueda \\ eufemiamr2@gmail.com \\ https://orcid.org/0000-0001-5265-0740 \\ Universidad César Vallejo. Lima - Perú \\ José Mercedes Vidal Sevilla \\ jomevise@gmail.com \\ https://orcid.org/0000-0001-7005-1557 \\ Universidad César Vallejo. Lima - Perú
}

\section{RESUMEN}

Los hábitos de lectura optimizan el aprendizaje de los estudiantes de primaria mejorando su rendimiento escolar, por ello el objetivo del presente artículo fue establecer la influencia que existe entre los hábitos de lectura y el aprendizaje de los estudiantes de primaria, El Agustino 2020. Lima - Perú. La metodología empleada fue bajo un enfoque cuantitativo, con un análisis correlacional - causal, de corte transversal. La muestra estuvo constituida por 86 estudiantes de los niveles de $5^{\circ}$ y $6^{\circ}$ grado de primaria de una población de 110 estudiantes. Para la medición se aplicaron dos cuestionarios validados por expertos. Como resultado se obtuvo que el coeficiente de correlación fue de - ,123, y una significancia bilateral de 0,261 mayor a 0,05 entre las dos variables de estudio por lo que se infiere que existe una relación escasa inversa entre las variables y no hay evidencia suficiente que relacione de forma significativa hábitos de lectura y aprendizaje.

Palabras clave: hábitos de lectura; aprendizaje; comprensión lectora; recursos; estudiantes. 


\title{
Reading habits and their influence on the learning of elementary school students
}

\begin{abstract}
Reading habits optimize the learning of elementary school students by improving their school performance, therefore the objective of this article was to establish the influence that exists between reading habits and learning of elementary school students, El Agustino 2020. Lima - Peru. The methodology used was under a quantitative approach, with a cross-sectional causal correlational analysis. The sample consisted of 86 students at the 5th and 6th grade levels of primary school out of a population of 110 students. For the measurement, two questionnaires validated by experts were applied. As a result, it was obtained that the correlation coefficient was,- 123 , and a bilateral significance of 0.261 greater than 0.05 between the two study variables, which is why it is inferred that there is a scarce inverse relationship between the variables and there is no evidence enough that it significantly relates reading and learning habits.
\end{abstract}

Keywords: reading habits; learning; reading comprehension; means; students.

Artículo recibido: 05 octubre. 2021 Aceptado para publicación: 02 noviembre 2021 Correspondencia: carlossevillam67@gmail.com Conflictos de Interés: Ninguna que declarar 


\section{INTRODUCCIÓN}

En la actualidad, el hábito lector y su impacto en el aprendizaje de los estudiantes en el Perú, es muy preocupantes según el Consejo Nacional de Educación (CNE) en el 2019 el promedio de ciudadanos que leen es de 0.86 , es decir que se lee menos de un libro por persona al año. Esta realidad moviliza a buscar posibles soluciones y ampliar las posibilidades para motivar la pasión por la lectura.

En este contexto Cases (2016), afirma que la lectura es esencial para la formación integral del alumnado, y la adquisición de un hábito lector es decisiva para su desarrollo educativo. Asimismo, Serna et al. (2017) señalan que para ello tiene que haber voluntad, ganas de leer y mucho interés. Por otro lado, Vásquez et al. (2018) reiteran que la realidad establece que es una habilidad, en muchas ocasiones, no desarrollada, practicada o aprovechada como se esperaría.

Paredes y Campo (2018) sostiene que Las dificultades en la comprensión de textos son por la ausencia de prácticas de lectura en los hogares de los escolares. Del mismo modo, Cerdán y Tovar (2018) indican que son los padres de familia quienes tienen la responsabilidad para motivar la práctica de lectura en el hogar y crear un ambiente propicio para la formación de un estudiante lector.

Por otro lado, los estudiantes que no reciben las estrategias adecuadas asumen un mal hábito y no siguen un proceso técnico de lectura (Cando et al., 2018), por eso, es importante que los docentes utilicen estrategias didácticas que mejoren la comprensión lectora para hacer estudiantes más competentes (Marín, 2019) una estrategia es el rincón de lectura que ayuda en la formación de valores (Mayorga, 2019); también la lectura en voz alta influye significativamente en la comprensión de textos (Jacinto et al., 2018); y la ejecución de programas de lectura (Olaya, 2019) apoyado en el enfoque interactivo impacta significativamente en la optimización de la comprensión lectora (Navarro, 2018); todas estas actividades de animación promueven el hábito lector en los estudiantes (Palacios, 2016). Asimismo, los lectores están vinculados a la literatura con diversas características sociales (Fraga, 2019); y basada en diferentes géneros literarios como la poesía, religión, libros juveniles, reportajes, novelas y ciencia ficción (Ortega, 2018)

En ese contexto, en la institución educativa No 046 Los Libertadores de Ayacucho se observa que los estudiantes de nivel primaria carecen de prácticas lectoras y que influye 
en su aprendizaje en todas las áreas del currículo escolar, esto se vuelve crítico porque hay una ausencia de soporte en los padres de familia que no refuerzan esta costumbre por leer en casa; por lo tanto, si los estudiantes no aprenden hábitos de lectura su aprendizaje corre el riesgo de ser lento y deficiente y como consecuencia tendrán bajo rendimiento escolar. Por ello el siguiente trabajo de investigación pretende contribuir a la mejora del problema a partir de la relación que existe entre los hábitos de lectura y el aprendizaje.

La investigación se justifica porque se basa en la revisión y dimensiones que forman parte de ese estudio y que aportará evidencias que permitirán comprender la problemática estudiada y seleccionar las mejores alternativas de solución.

En tal sentido el objetivo principal de esta investigación fue establecer la influencia que existe entre los hábitos de lectura y el aprendizaje de los estudiantes de $5^{\circ}$ y $6^{\circ}$ grado de primaria de la IE Nº 046, Libertadores de Ayacucho, El Agustino.

\section{ESTRATEGIAS METODOLÓGICAS O MATERIALES Y MÉTODOS}

La investigación realizada fue de enfoque cuantitativo y tuvo un alcance de tipo correlacional - causal porque se analizó la relación entre los hábitos de lectura y aprendizaje. La investigación se realizó en el periodo de abril y agosto del 2020 con una población de estudio es de 110 escolares de 5to y 6to grado de primaria de la IE No 046, Los Libertadores de Ayacucho; en la que se consideró las características comunes o específicas de la población mediante el muestreo probabilístico (Hernández et al., 2018) y la muestra representativa fue de 86 estudiantes seleccionados en forma aleatoria.

Como instrumento de recolección de datos se utilizaron dos instrumentos los cuales fueron validados por tres expertos. Los cuestionarios fueron elaborados sobre una base de preguntas cerradas y aplicado a los estudiantes de 5to y 6to grado de la IE $\mathrm{N}^{\circ} 046$ Los Libertadores de Ayacucho, para así conocer el nivel de relación entre las variables y sus dimensiones. El cuestionario de la variable independiente: Hábitos de lectura, estuvo compuesta por 4 dimensiones: tiempo, motivación, recursos y metodología con un total de 16 ítems y una escala de valor ordinal de siempre (5), casi siempre (4), a veces (3), Casi nunca (2) nunca (1). e igualmente el cuestionario de la variable dependiente: Aprendizaje, estuvo compuesta por 5 dimensiones: aptitudes y percepciones, adquirir e integrar el conocimiento, extender y refinar el conocimiento y uso significativo del conocimiento y hábitos mentales con un total de 25 ítems y con 
valores ordinales de siempre (5), casi siempre (4), a veces (3), Casi nunca (2) nunca (1). Para obtener el grado de confiabilidad se realizó el análisis de consistencia interna utilizando el método de coeficiente Alfa Cronbach. La variable "hábitos de lectura" obtuvo el valor de alfa de Cronbach 0,780 , calificando como moderado. La variable "aprendizaje" obtuvo el valor de alfa de Cronbach 0,907, que lo calificó como muy satisfactoria, pasando así la prueba de fiabilidad, lo que significó que los instrumentos eran confiables.

El procedimiento de recolección de datos se realizó sensibilizando a cada estudiante, para luego aplicar los cuestionarios y que sean respondidos por los escolares contando con un tiempo de duración de 25 minutos para el cuestionario de la variable "hábitos de lectura" y 35 minutos para "Aprendizaje".

Para el procesamiento de datos se utilizó el programa Excel 2016; y luego de haber determinado los rangos de cada una de las variables y dimensiones se utilizó el programa estadístico SPSS versión 26. El análisis descriptivo se realizó usando frecuencias y porcentajes de las variables individuales y tabulación cruzada por asociación. Para el análisis inferencial se aplicó la regresión ordinal simple a través del coeficiente de correlación de Spearman que permitió contrastar la hipótesis, considerando los parámetros a un nivel de confianza del 95\% y significancia del $5 \%$.

De acuerdo a las características del estudio se tomó en cuenta los aspectos éticos, solicitando permiso al director, profesores y autorización de los padres de familia haciendo uso del consentimiento informado para aplicar la encuesta a los estudiantes; la información recogida, guardo el principio de confidencialidad y se utilizó exclusivamente para para el objetivo de la investigación.

\section{RESULTADOS}

La tabla 1 destaca los resultados de la encuesta acerca de los hábitos de lectura de los estudiantes de la institución educativa No 046 Los Libertadores de Ayacucho mostrando que el 87,2\% (75) de los estudiantes encuestados indicó que la frecuencia de los hábitos de lectura es de nivel logrado, mientras que un 12,8\% (11) opinó que son de un nivel de proceso. Observándose la predominancia del nivel logrado. 


\section{Tabla 1}

Hábitos de lectura

\begin{tabular}{llc|c|c|c} 
& & Frecuencia & Porcentaje & $\begin{array}{c}\text { Porcentaje } \\
\text { válido }\end{array}$ & $\begin{array}{c}\text { Porcentaje } \\
\text { acumulado }\end{array}$ \\
\hline \multirow{3}{*}{ Válido } & Proceso & 11 & 12,8 & 12,8 & 12,8 \\
\cline { 2 - 7 } & Logrado & 75 & 87,2 & 87,2 & 100,0 \\
\cline { 2 - 7 } & Total & $\mathbf{8 6}$ & $\mathbf{1 0 0 , 0}$ & $\mathbf{1 0 0 , 0}$ & \\
\hline
\end{tabular}

Fuente: Elaboración propia.

La tabla 2 muestra que el resultado de la frecuencia de aprendizaje fue de 90,7 \% (78) de nivel de logrado, mientras que el 9,3\% (8) fue de nivel proceso. Apreciándose que el nivel predominante fue de logrado.

\section{Tabla 1}

Aprendizaje

\begin{tabular}{|c|c|c|c|c|c|}
\hline & & Frecuencia & Porcentaje & $\begin{array}{l}\text { Porcentaje } \\
\text { válido }\end{array}$ & $\begin{array}{l}\text { Porcentaje } \\
\text { acumulado }\end{array}$ \\
\hline \multirow{3}{*}{ Válido } & Proceso & 8 & 9,3 & 9,3 & 9,3 \\
\hline & Logrado & 78 & 90,7 & 90,7 & 100,0 \\
\hline & Total & 86 & 100,0 & 100,0 & \\
\hline
\end{tabular}

Fuente: Elaboración propia

Con relación a la tabla 3 se halló que los niveles de asociación fueron de mayor porcentaje en un nivel logrado tanto para hábitos de lectura y aprendizaje en un $77,9 \%$ y el más bajo porcentaje fue de $0 \%$ para el nivel de proceso en ambas variables.

\section{Tabla 3}

Tabla cruzada Hábitos de lectura*Aprendizaje.

\begin{tabular}{lllccc}
\hline & & & \multicolumn{2}{c}{ Aprendizaje } & \multirow{2}{*}{ Total } \\
\cline { 3 - 5 } & & & Proceso & Logrado & \\
\hline \multirow{3}{*}{ Hábitos de lectura } & Proceso & Recuento & 0 & 11 & 11 \\
& & $\%$ del total & $0,0 \%$ & $12,8 \%$ & $12,8 \%$ \\
\cline { 2 - 5 } & \multirow{2}{*}{ Logrado } & Recuento & 8 & 67 & 75 \\
& & $\%$ del total & $9,3 \%$ & $77,9 \%$ & $87,2 \%$ \\
\hline \multirow{2}{*}{ Total } & & Recuento & 8 & 78 & 86 \\
& & $\%$ del total & $9,3 \%$ & $90,7 \%$ & $100,0 \%$ \\
\hline
\end{tabular}

Fuente: Elaboración propia 
De acuerdo con la prueba de inferencia se obtuvo que el coeficiente de correlación entre hábitos de lectura y aprendizaje fue de -,123, por lo que se infiere que existe una asociación escasa e inversa entre las variables estudiadas entre las variables estudiadas y se obtuvo un sigma bilateral de $\mathrm{p}=0,261$ mayor a 0,05 , por lo cual se acepta la hipótesis nula y se rechaza la hipótesis alterna, infiriendo que los hábitos de lectura no influye en el aprendizaje de los estudiantes de la institución educativa 046 de El Agustino (Tabla 4)

\section{Tabla 4}

Correlación de Spearman de las variables: Hábitos de lectura y aprendizaje

\begin{tabular}{|c|c|c|c|c|}
\hline & & & $\begin{array}{c}\text { Hábitos de } \\
\text { lectura }\end{array}$ & Aprendizaje \\
\hline \multirow{6}{*}{ Rho de Spearman } & \multirow{3}{*}{ Hábitos de lectura } & $\begin{array}{l}\text { Coeficiente de } \\
\text { correlación }\end{array}$ & 1,000 &,- 123 \\
\hline & & Sig. (bilateral) & . & ,261 \\
\hline & & $\mathrm{N}$ & 86 & 86 \\
\hline & \multirow{3}{*}{ Aprendizaje } & $\begin{array}{l}\text { Coeficiente de } \\
\text { correlación }\end{array}$ &,- 123 & 1,000 \\
\hline & & Sig. (bilateral) & ,261 & . \\
\hline & & $\mathrm{N}$ & 86 & 86 \\
\hline
\end{tabular}

Fuente: Elaboración propia

\section{DISCUSIÓN}

El objetivo de la investigación fue establecer la influencia que existe entre los hábitos de lectura y el aprendizaje de los estudiantes de primaria, El Agustino.

Los resultados muestran que la relación existente entre los hábitos de lectura y el aprendizaje de los estudiantes de primaria, El Agustino tiene una correlación de - 0,123; con un $\mathrm{p}=$,261 mayor a 0,05 lo que indica una relación escasa inversa entre las variables y que no hay vínculo representativo entre hábitos de lectura y aprendizaje; por lo que es necesario mejorar las estrategias de lectura para mejorar el aprendizaje. Estos resultados se relacionan con los que encontraron Cerdán y Tovar (2018); que la carencia de hábitos de lectura incide en el bajo rendimiento escolar. Por otro lado, Vásquez, et al. (2018) sostiene que los profesores dan por hecho que la lectura y la comprensión son temas dominados por los estudiantes, sim embargo la realidad establece que es una habilidad, que en muchas ocasiones no está desarrollada como se esperaría. 
Así mismo al establecer la relación entre las variables tiempo y aprendizaje los resultados mostraron una relación con $\mathrm{r}=-0,015$ y un nivel de significancia de $\mathrm{p}=0,892$ indicando una relación escasa inversa lo que evidenció que no hay relación significativa entre las dos variables por lo que es importante optimizar los horarios de lectura. Estos resultados se asemejan a lo que manifiestan Paredes y Campo (2018) que los problemas en la comprensión de textos, la expresión oral y escrita es porque no se proporciona tiempo para la lectura y escritura en los hogares escolares, además el bajo nivel académico de muchos padres y sujetos cercanos a los niños no es suficiente para brindar un acompañamiento educativo que necesitan los niños para su desarrollo integral.

Por otro lado, al establecer la relación entre motivación y aprendizaje, los resultados estadísticos fueron de una correlación de $r=-0,147$ y una significancia de $p=0,176$ expresando una relación escasa inversa lo que indicó que no hay relación significativa entre las dos variables. Estos resultados concuerdan con Ortega, (2018) que concluyó en su investigación que la motivación por la lectura era de iniciativa propia. Situación que reafirma Palacios (2016) que las actividades de animación promueven el hábito lector en los estudiantes.

Así mismo, al establecer la relación las variables recursos y aprendizajes, los resultados presentaron una relación con $\mathrm{r}=-0,109$ y una $\mathrm{p}=0,316$ mayor a 0,05 ; lo que expresa que no hay vinculo representativo entre las dos variables. A estos resultados, Ortega (2018) indica que para leer debe haber recursos y materiales de lectura basados en géneros literarios como la poesía, religión, libros juveniles, novelas y ciencia ficción; en esa misma línea, Fraga (2019) sostiene que los lectores están vinculados a la literatura con diversas características sociales.

En cuanto a la relación entre las variables metodología y aprendizaje, los resultados mostraron una relación con $\mathrm{r}=-0,023$ y una significancia de 0,831 lo cual expresa que no hay una relación significativa; resultado que difiere con Olaya (2019) que, sostiene que la aplicación estratégica programas de lectura aumenta representativamente la comprensión lectora de los estudiantes. Además, se relaciona con lo que refiere Marín (2019) que, es importante que los docentes utilicen estrategias didácticas que mejoren la comprensión lectora y Mayorga (2019) afirma que, una estrategia es el rincón de lectura que ayuda en la formación de hábitos lectores. 


\section{CONCLUSIÓN O CONSIDERACIONES FINALES}

Con respecto al objetivo general se concluye que los hábitos de lectura no se relacionan significativamente con el aprendizaje en los estudiantes de primaria, El Agustino. La relación es inversa y escasa, lo que significa que las estrategias de lecturas no han aportado al aprendizaje de los escolares y se necesita reformular las estrategias lectoras en la que tienen que tener mayor participación los padres y profesores para estimular, promover y despertar el interés en la adquisición del hábito de lectura y esta les ayude en su aprendizaje.

Asimismo, se concluyó que no se encontró relación significativa entre la variable aprendizaje en correspondencia con las variables, tiempo, motivación, recursos y metodología significa porque falta optimizar las estrategias de lectura para que los estudiantes se sientan motivados por leer y en consecuencia aprender. Asimismo, deben contar con recursos materiales como son libros, revistas, bibliotecas en casa y en el colegio, bibliotecas virtuales, pero que tienen que organizar y establecer horarios de lectura tanto en el hogar como en el colegio para incentivar el hábito de lectura y de esta forma mejorar sus aprendizajes

De todo lo presentado, se determinó que para considerar que los hábitos de lectura como un medio para que contribuya al aprendizaje de los escolares se tiene que sensibilizar a los agentes principales en los espacios de la escuela y el hogar, es decir, padres de familia y profesores para que cumplan su rol de manera responsable; motivando, brindando los recursos, organizando el tiempo y estableciendo una metodología para lograr los objetivos propuestos.

\section{LISTA DE REFERENCIAS}

Cando, M., Chuquimarca, D., Pazmuño, L. (2018). Influencia del hábito de la lectura en la enseñanza aprendizaje del vocabulario en estudiantes de octavo año de educación básica. Erupoean Scientific Journal 14(22). 187-201 http://dx.doi.org/10.19044/esj.2018.v14n22p187

Cases, I (2016) El hábito lector en alumnos de Educación Primaria. (Tesis de maestría Universidad Illes Ballears, Baleares, España) https://dspace.uib.es/xmlui/bitstream/handle/11201/3438/Cases_Soler_Ines.pdf? sequence $=1 \&$ isAllowed $=y$ 
Cerdán, J., y Tovar, D. (2018). Hábitos de lectura de los estudiantes del VII ciclo de la institución educativa Francisco Bolognesi-Tantará. (Tesis segunda especialidad Universidad Nacional de Huancavelica). https://bit.ly/312xYK1

Fraga, J. (2019). En busca del lector: una mirada a los hábitos de lectura en España. (Tesis de maestría). Universidade Da Coruña, Coruña, España. http://hd 1.handle.net/2183/23608

Jacinto, L., Landa, R., López, C. (2018). La lectura en voz alta como estrategia metodológica para mejorar la comprensión lectora en estudiantes del $3^{\circ}$ grado Educación Primaria de la I.E. José Olaya Balandra 6090 - Chorrillos. (Tesis maestría Universidad Nacional Enrique Guzmán y Valle) http://repositorio.une.edu.pe/handle/UNE/1928

Hernández, R. Fernández, C. y Baptista, P. (2018). Metodología de la investigación científica. McGraw-Hill Education. México.

Marín, I. (2019). Estrategias didácticas utilizadas en la enseñanza de la comprensión y análisis de la lectura en estudiantes de primaria. (Tesis de Maestría Universidad de Montemorelos, Matamoros, México).

http://dspace.biblioteca.um.edu.mx/xmlui/bitstream/handle/20.500.11972/1044/ TESIS\%20\%20IRIS\%20MARIN.pdf?sequence $=1 \&$ isAllowed $=\mathrm{y}$

Mayorga, N. (2019). El rincón de lectura y su influencia en el aprendizaje de los valores de los niños de 5 a 6 años de la unidad educativa Cayetano Tarruell. (Tesis de licenciatura Universidad Laica Vicente Rocafuerte de Guayaquil). http://repositorio.ulvr.edu.ec/bitstream/44000/3425/1/T-ULVR-3005.pdf

Navarro, L. (2018). Programa de lectura basado en el enfoque interactivo para mejorar la comprensión de textos en los estudiantes de $4^{\circ}$ grado de educación primaria de la I.E. República Argentina N80008 Trujillo 2017. (Tesis de maestría Universidad Católica Los Ángeles)

http://repositorio.uladech.edu.pe/handle/123456789/10230

Olaya, R. (2019). Efectos del programa estrategias cognitivas y metacognitivas sobre la comprensión lectora en estudiantes de una institución educativa pública. (Tesis de maestría Universidad Marcelino Champagnat) http://repositorio.umch.edu.pe/handle/UMCH/567 
Ortega, L. (2018). Hábitos de interés por la lectura que tienen los alumnos de la Escuela Normal Intercultural de Chiquimula. (Tesis de licenciatura Universidad Rafael Landívar, Guatemala).

http://recursosbiblio.url.edu.gt/tesiseortiz/2018/05/09/Ortega-Lourdes.pdf

Palacios, M. (2015), Fomento del hábito lector mediante la aplicación de estrategias de animación a la lectura en primero de secundaria. (Tesis de maestría Universidad de Piura) https://pirhua.udep.edu.pe/bitstream/handle/11042/2301/MAE_EDUC_161.pdf

Paredes, J., Campo, M. (2018). La lectura como herramienta de estimulación en el rendimiento escolar de los estudiantes de segundo grado de la I.E.D rural El Altico sede Rincón Santo. (Tesis de licenciatura Corporación Universitaria minuto de Dios, Colombia).

https://repository.uniminuto.edu/bitstream/handle/10656/7628/T.EDI_ParedesCo ntrerasJessicaTatiana 2018.pdf? sequence $=1$ \&isAllowed $=y$

Serna, M., Rodríguez, A., y Etxaniz, X. (2017). Biblioteca escolar y hábitos lectores en los escolares de Educación Primaria. Revista Ocnos. 16 (1), 18-49. http://dx.doi.org/10.18239/ocnos_2017.16.1.1205

Tello, A. (23 de abril de 2021). Día del Libro 2021: convierte la lectura en un hábito. Métrica. https://metrica.pe/blog/dia-del-libro-2021-convierte-la-lectura-en-unhabito

Vásquez, E., Gazca, S., y Méndez, R. (2018). Influencia de la lectura en el rendimiento académico de estudiantes del campus de ciencias exactas e ingenierías de la Universidad Autónoma de Yucatán. Ingeniería, 23 (2),1-26.

http://www.revista.ingenieria.uady.mx/ojs/index.php/ingenieria/article/view/128 